\title{
Conference on In Situ and Correlative Electron Microscopy (CISCEM 2016)
}

Niels de Jonge ${ }^{1,2}$, Kristian Mølhave ${ }^{3}$ and Damien Alloyeau ${ }^{4}$

${ }^{1 .}$ INM - Leibniz Institute for New Materials, 66123 Saarbrücken, Germany.

2. Department of Physics, University of Saarland, 66123 Saarbrücken, Germany.

3. Dept. of Micro and Nanotechnology, Technical University of Denmark (DTU), Denmark.

4. Laboratoire Matériaux et Phénomènes Quantiques, Université Paris 7 - CNRS, Paris, France.

CISCEM2016 was held from 11-12 Oct. 2016 in Saarbrücken, Germany. This conference brought together an interdisciplinary group of scientists from the fields of biology, materials science, geology, chemistry, and physics, to discuss future directions of in situ electron microscopy research. Topics included nanoscale studies of biological samples, and functional materials under realistic or near realistic conditions, for example, in gaseous environments, at elevated temperatures, and in liquid. It was shown how dynamical processes are studied by including the time domain in electron microscopy, while taking into account the electron beam effects. CISCEM was also open to other in situ techniques, such as X-ray, near field or scanning probe microscopy, with the view to stimulate fruitful discussions on multi-scale and correlative approaches. Members of the scientific organizing committee were Niels de Jonge, Kristian Mølhave, and Damien Alloyeau. This conference was organized for the third time at the venue of the campus of the Saarland University.

Highlight was the keynote lecture of Dr. Frances M. Ross, IBM, Yorktown Heights, NY, USA, who spoke about liquid-cell transmission electron microscopy for imaging electrochemical processes. The other invited speakers were:

- Prof. Ovidiu Ersen, IPCMS, Université de Strasbourg, France

- Dr. Layla Mehdi, Pacific Northwest National Laboratory, Richland, WA, USA

- Dr. Jacob Hoogenboom, Delft University of Technology, Netherlands

- Dr. Matthia Karreman, EMBL European Molecular Biology Laboratory, Heidelberg, Germany

- Prof. Bram Koster, Leiden University Medical Center, Netherlands

- Prof. Utkur Mirsaidov, National University of Singapore, Singapore

- Dr. Diana Peckys, Saarland University, Saarbrücken, Germany

- Dr. Tanya Prozorov, Ames Laboratory, IA, USA

- Dr. Chongmin Wang, Pacific Northwest National Laboratory, Richland, WA, USA

- Dr. Marc Willinger, Fritz Haber Institute of the Max Planck Society, Berlin, Germany

The abstracts published here reflect the interdisciplinary nature of the CISCEM conference that covered seven thematic sessions:

1) Studying reactions at the solid-gas interface with environmental electron microscopy. Both environmental transmission electron microscopy (ETEM) and environmental scanning electron microscopy (ESEM) are providing unique new insights into the complex reaction dynamics, and getting ever closer to in operando conditions [01-2]. One of the newest advances in this area involves rapid ETEM tomography aiming for three-dimensional in situ microscopy [O1-3].

2) Liquid-phase and correlative electron microscopy of soft matter and biomaterials. Liquid cell TEM provides direct imaging studies of biomineralization processes and self-assembly processes with block copolymers [O2-2], and explorative studies of structures in bacteria [O2-1].

3) In situ electron microscopy of electrochemical processes. This field is rapidly growing as was 
reviewed by Frances Ross [O3-1]. Many fundamental aspects of radiolysis and optimization of the methods are ongoing. Insight into delicate details of, for instance, battery electrode processes have been achieved both by open cell system using ionic liquids [O3-2], and using closed cells with regular liquids [O3-3].

4) Liquid phase and correlative electron microscopy of cells and proteins. The complex nature of biological structures and processes with limited contrast in electron microscopy pose a challenging system for liquid-cell studies. New approaches are being explored for membrane proteins in cells [O4-1], and vesicles [O4-4]. Correlative microscopy approaches are increasingly powerful to provide dynamic and detailed information about processes [O4-2 and O4-3].

5) Liquid-phase electron microscopy of nanomaterials. The fundamental aspects of nanoparticle nucleation and growth are studied with liquid-cell TEM for gold nanoparticles interactions [O51], faceted growth [O5-3], and magnetic nanoparticles [O5-2].

6) Innovations in methods for correlative microscopy. Correlating methods in materials science provides a more complete picture of phenomena than achievable with one technique only. Superresolution fluorescence microscopy can now be done in SEM [O6-1]. Correlating electron microscopy with x-ray methods [O6-3], and with three dimensional atom probe imaging [06-4] open up new possibilities.

7) In situ measurements of materials properties. Actively probing mechanical [O7-1], electrical [07-2, O7-3], and optical [07-2] properties using in situ electron microscopy provides direct correlation between quantitative measurements and observed morphological changes.

The poster pitch selected three posters for brief presentations demonstrated promising new approaches, such as electron holography on liquid samples [P5-2], atomic force microscopy on protein conformational changes [P3-3], and graphene membranes for liquid cells [P4-2].

Overall the wide variety of materials and dynamical phenomena investigated under realistic conditions demonstrates the rapidly growing interest of the international scientific community for in situ characterization at the nanometer length scale, transforming electron microscopes from merely imaging devices into full experimental platforms capable of multi-parameter quantitative exploration combined with (sub-) nanoscale observations of static- and changing structures.

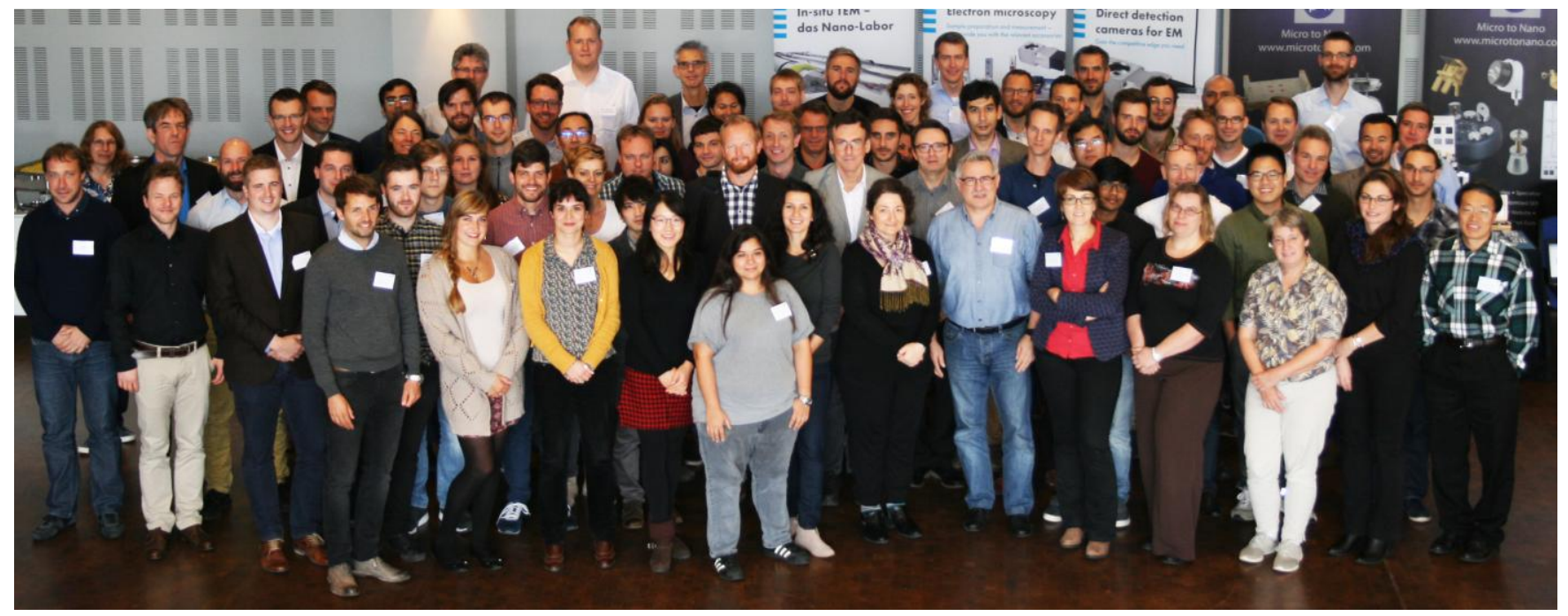

Figure 1. Group photo of CISCEM 2016 


\section{Acknowledgements}

Christine Hartmann and Dominique Hell organized the logistics of the conference, and edited the abstract booklet. The INM provided financial support through Eduard Arzt.

The following sponsors are greatly acknowledged:

- Carl Zeiss Microscopy, Oberkochen, Germany

- CEOS GmbH, Heidelberg, Germany

- European Microscopy Society

- Delmic, Delft, Netherlands

- DENSsolutions, Delft, Netherlands

- Deutsche Gesellschaft fuer Elektronenmikroskopie, Germany

- FEI, Hillsboro, OR, USA

- Gatan Inc., Pleasanton, CA, USA

- JEOL Ltd., Tokyo 196-8558, Japan

- NanoMEGAS, Forest, Belgium

- Nanosurf, Liestal, Switzerland

- MicroToNano, Haarlem, Netherlands

- Phenom World, Eindhoven, Netherlands

- Protochips, Inc., Raleigh, NC, USA

- LOT-QuantumDesign GmbH, Darmstadt, Germany

- WITec, Ulm, Germany 